\title{
Avaliação da capacidade para o trabalho dos profissionais da atenção primária à saúde: Estudo transversal
}

\author{
Evaluation of the work ability of primary health care professionals: A cross-sectional study \\ Evaluación de la capacidad laboral de los profesionales de atención primaria de salud: Un estudio \\ transversal
}

Recebido: 13/07/2021 | Revisado: 21/07/2021 | Aceito: 27/07/2021 | Publicado: 03/08/2021

Cassia Gonçalves Santos da Silveira

ORCID: https://orcid.org/0000-0002-7062-5298 Universidade Federal Fluminense, Brasil E-mail: cassiasilveira@id.uff.br

Barbara Pompeu Christovam

ORCID: https://orcid.org/0000-0002-9135-8379 Universidade Federal Fluminense, Brasil E-mail: barbarachristovam@id.uff.br

Josélia Braz dos Santos Ferreira

ORCID: https://orcid.org/0000-0001-7695-8598 Universidade Federal Fluminense, Brasil E-mail: joseliabraz42@yahoo.com.br

Selma Petra Chaves Sá

ORCID: https://orcid.org/0000-0001-9878-7179 Universidade Federal Fluminense, Brasil

E-mail: selmapetrasa@gmail.com

Ana Maria Fernandes Pitta

ORCID: https://orcid.org/0000-0003-2558-5757

Universidade Católica do Salvador, Brasil

E-mail: ana.maria.pitta@gmail.com

Jonas Lirio Gurgel

ORCID: https://orcid.org/0000-0002-9355-7793

Universidade Federal Fluminense, Brasil

E-mail: jonasgurgel@id.uff.br

\begin{abstract}
Resumo
Objetivou-se avaliar a capacidade funcional dos profissionais da Estratégia de Saúde da Família por meio do Índice de Capacidade para o Trabalho. Trata-se de estudo transversal, exploratório, descritivo, utilizado como instrumento de análise, a escala Índice de Capacidade para o Trabalho. A pesquisa foi realizada em nove unidades de Atenção Básica de Saúde do Rio de Janeiro, Brasil, de julho a novembro de 2018, com 88 profissionais de equipes multiprofissionais. Utilizou-se estatística descritiva a todas as variáveis e cálculo de coeficiente de variação para as variáveis quantitativas. A maioria dos participantes é do gênero feminino (80,7\%), com média de idade de 43 anos (40,6\%), trabalhando atualmente em apenas um emprego $(85,2 \%)$ e com uma carga horária semanal de trabalho de 40 horas $(87,5 \%)$ e estando trabalhando no atual local de trabalho há apenas um ano (30,7\%). Sentiam-se exigidos no trabalho física e mentalmente $(95,5 \%)$ profissionais. As comorbidades mais prevalentes foram: hipertensão arterial e lesões nas costas, ambas com $(26,1 \%)$ participantes. A pesquisa também mostrou que doenças relacionadas com lesões nas costas ( $\mathrm{p}$-valor $=0,013)$ e depressão leve ( $\mathrm{p}$-valor $=0,002)$ influenciaram diretamente na capacidade para o trabalho, e repercutiram em escores mais baixos, que foram confirmados pelo teste estatístico de Mann Whitney. Concluiu-se que dores musculoesqueléticas e depressão leve diminuíram a capacidade para o trabalho dos profissionais de saúde da atenção básica. Este estudo busca contribuir para pesquisas na temática da saúde do trabalhador, no acompanhamento da capacidade funcional dos profissionais de saúde, visando a prevenção de doenças ocupacionais e o processo de envelhecimento saudável.
\end{abstract}

Palavras-chave: Atenção primária à saúde; Saúde do trabalhador; Trabalhadores; Política pública; Aposentadoria.

\begin{abstract}
The objective was to assess the functional capacity of professionals in the Family Health Strategy through the Work Ability Index. This is a cross-sectional, exploratory, descriptive study, used as an instrument of analysis, using the Work Ability Index scale. The survey was conducted in nine Primary Health Care units in Rio de Janeiro, Brazil, from July to November 2018, with 88 professionals from multidisciplinary teams. Descriptive statistics were used for all variables and coefficient of variation calculation for quantitative variables. Most participants are women (80.7\%), with an average age of 43 years $(40.6 \%)$, currently working in just one job (85.2\%) and with a weekly workload of 40
\end{abstract}


hours $(87.5 \%)$ and having been working in the current workplace for just one year (30.7\%). They felt demanded to work physically and mentally $(95.5 \%)$ professionals. The most prevalent comorbidities were: hypertension and back injuries, both with $(26.1 \%)$ participants. The research also showed that illnesses related to back injuries (pvalue $=0.013)$ and mild depression $(p$-value $=0.002)$ directly influenced work ability, and reflected in lower scores, which were confirmed by the statistical test of Mann Whitney. It was concluded that musculoskeletal pain and mild depression decreased the work capacity of primary care health professionals. This study seeks to contribute to research on the theme of workers' health, in monitoring the functional capacity of health professionals, aiming at the prevention of occupational diseases and the healthy aging process.

Keywords: Primary health care; Worker's health; Workers; Public policy; Retirement.

\section{Resumen}

El objetivo fue evaluar la capacidad funcional de los profesionales de la Estrategia Salud de la Familia a través del Índice de Capacidad Laboral. Se trata de un estudio descriptivo, exploratorio, transversal, utilizado como instrumento de análisis mediante la escala Work Ability Index. La investigación se realizó en nueve unidades de Atención Primaria de Salud en Río de Janeiro, Brasil, de julio a noviembre de 2018, con 88 profesionales de equipos multidisciplinarios. Se utilizó estadística descriptiva para todas las variables y cálculo del coeficiente de variación para las variables cuantitativas. La mayoría de los participantes son mujeres (80,7\%), con una edad media de 43 años $(40,6 \%)$, actualmente trabajan en un solo empleo $(85,2 \%)$ y con una carga de trabajo semanal de 40 horas $(87,5 \%)$ y que han estado trabajando en lo mismo lugar de trabajo solamente un año $(30,7 \%)$. Se sintieron obligados a trabajar física y mentalmente $(95,5 \%)$ profesionales. Las comorbilidades más prevalentes fueron: hipertensión y lesiones de espalda, ambas con $(26,1 \%)$ participantes. La investigación también mostró que las enfermedades relacionadas con las lesiones de espalda (valor $p=0,013$ ) y la depresión leve (valor $p=0,002$ ) influyeron directamente en la capacidad para el trabajo, y se reflejaron en puntuaciones más bajas, que fueron confirmadas por la prueba estadística de Mann Whitney. Se concluyó que el dolor musculoesquelético y la depresión leve disminuyeron la capacidad de trabajo de los profesionales de la salud de atención primaria. Este estudio busca contribuir a la investigación sobre el tema de la salud de los trabajadores, en el seguimiento de la capacidad funcional de los profesionales de la salud, con el objetivo de la prevención de enfermedades profesionales y el proceso de envejecimiento saludable.

Palabras clave: Atención primaria de salud; Salud del trabajador; Trabajadores; Política pública; Jubilación.

\section{Introdução}

A avaliação da saúde do trabalhador quanto à sua capacidade para o trabalho mostra-se relevante, pois o profissional necessita ser avaliado e acompanhado, objetivando à prevenção de doenças ocupacionais, que podem aparecer ao longo de sua função laboral e se acentuar com o processo de envelhecimento e com o aparecimento de doenças crônicas (Martins, Abreu, Silva \& Lima, 2020).

A questão do envelhecimento e o potencial dos idosos constitui sólida base para o desenvolvimento futuro e esta permite à sociedade recorrer cada vez mais à competência, experiência e sabedoria dos idosos, não só para tomar a iniciativa de sua própria melhoria, mas também para participar ativamente de toda a sociedade (ONU, 2003). O processo de envelhecimento depende de muitos fatores intrínsecos e extrínsecos de cada indivíduo (Mari, Alves, Aerts \& Câmara, 2016).

Assim, em relação à avaliação dos profissionais de saúde e trabalhadores de um modo geral, considerando-se a forma como estão envelhecendo, torna-se imprescindível adotar um instrumento de avaliação conforme o Índice de Capacidade para o Trabalho (ICT) é relevante quanto às propriedades psicométricas. Um este estudo brasileiro com 1.436 trabalhadores de enfermagem de três hospitais públicos do Rio de Janeiro, comprovou as hipóteses teóricas da validade relacionadas à correspondência e importância do ICT com os escores de recompensa, controle e autoavaliação relacionado à saúde e a necessidade de descanso após excesso de esforço devido às demandas do trabalho. Dessa maneira, o ICT avalia a percepção do trabalhador o quanto está a capacidade para o trabalho neste momento atual, ou quão estará em um futuro próximo, e quão este trabalhador pode realizar seu trabalho, em conformidade com as condições impostas, de seu estado de saúde e capacidades físicas e mentais (Silva Junior, Vasconcelos, Griep \& Rotenberg, 2011).

Segundo Bravo (2009), a saúde nasce vinculada ao mundo do trabalho, pois os trabalhadores reivindicavam seus direitos diante das condições de trabalho. Um passo importante a quaisquer reivindicações é um conhecimento aprofundado 
dos fatores que influenciam as condições de saúde dos trabalhadores bem como as próprias condições de trabalho as quais estes são submetidos.

A avaliação dos profissionais visando qualidade de vida e o direito a condições dignas e acesso ao trabalho torna-se ainda mais necessária em vigência da reforma da previdência, e que fez recair sobre o trabalhador, o aumento do tempo de serviço e consequentemente, o aumento do período de contribuição previdenciária, conforme dados divulgados pelo Instituto Nacional do Seguro Social - INSS, em 18 de novembro de 2019, quando pontua as principais mudanças realizadas a nível nacional (INSS, 2019).

As mudanças observadas no perfil demográfico e epidemiológico da população brasileira têm acompanhado as transformações políticas, sociais e econômicas, às quais a sociedade tem passado ao longo dos anos. Essas mudanças se caracterizam por um processo contínuo e intenso do envelhecimento. Contudo, a insuficiência ou até mesmo a falta de implementação de ações sistematizadas que incluem avaliação e monitoramento de fatores de risco preveníveis voltadas ao envelhecimento saudável, e conforme o modelo vigente de organização dos Serviços de Saúde em Redes de Atenção à Saúde (RAS), ainda se mostram incipientes (Brasil, 2018; Brasil, 2006).

Guardadas as especificidades laborais, o envelhecimento dos profissionais da equipe de saúde transcorre como nas demais profissões, e com a atual política trabalhista adotada no Brasil, que aponta a reforma na previdência, em que aumenta o tempo de contribuição e consequentemente da idade para aposentadoria (INSS, 2019). Acredita-se que este fato possa trazer implicações sérias à saúde destes profissionais bem como no desempenho das atividades laborais, às quais se voltam à prestação de cuidados de diferentes níveis de complexidade à população.

A identificação precoce de situações e de fatores relacionados ao trabalho que possam afetar as condições de saúde dos trabalhadores que exercem suas atividades na APS, e pode propiciar as definições de ações e estratégias de implementação de medidas preventivas bem como a promoção, recuperação da saúde e da capacidade laboral, por parte de gestores e profissionais. Considera-se que o acompanhamento da saúde dos trabalhadores que atuam na atenção básica pode estar aquém do ideal e que medidas preventivas poderão melhorar a capacidade para o trabalho desses profissionais.

O interesse do desenvolvimento do presente estudo advém do exercício profissional dos autores, em que puderam observar que o envelhecimento humano, que é referenciado como um fenômeno de ordem mundial, encontra-se presente na equipe de saúde, cujo fenótipo observável relacionado aos cabelos brancos não são os seus principais indicativos, mas sim a progressão das limitações de ordem física ou cognitiva, que requisitam mais atenção, por tratar-se de pessoas que cuidam da saúde de outras pessoas. Tais considerações descritas têm como ponto de partida proceder a uma avaliação sobre as condições de saúde dos profissionais atuantes na atenção primária de saúde, pela necessidade de implementar ações sistematizadas de avaliação, monitoramento e intervenção das condições de saúde, que propiciem qualidade de vida e índices adequados de capacidade para o trabalho desses profissionais no contexto do trabalho. No entanto, a avaliação da capacidade para o trabalho de profissionais de saúde da Atenção Primária à Saúde (APS), apresentou-se com lacuna de conhecimento, escassez de produções científicas no tema saúde do trabalhador, principalmente na esfera da Estratégia de Saúde da Família (ESF). Assim, o desenvolvimento deste estudo se torna relevante. Logo, se estabeleceu a seguinte questão norteadora: Que fatores estão consideravelmente associados à capacidade funcional dos profissionais de saúde da Atenção Primária à Saúde?

Neste contexto, o objetivo deste estudo consistiu em avaliar a capacidade funcional dos profissionais da Estratégia de Saúde da Família por meio do Índice de Capacidade para o Trabalho.

\section{Metodologia}

Trata-se de estudo transversal, exploratório, descritivo, de abordagem quantitativa. O estudo transversal viabiliza a determinação simultânea entre o fator de interesse e o desfecho em uma investigação, e realizada em uma população bem 
definida em determinado momento (Gil, 2002). A pesquisa de caráter quantitativo permite representar, quantitativamente, as relações entre os eventos.

Cabe lembrar que o presente estudo seguiu as diretrizes da iniciativa Strengthening the Reporting of Observational Studies in Epidemiology - STROBE (STROBE, 2007). Os itens que compõem o STROBE são relacionados às informações que devem estar presentes no título, resumo, introdução, metodologia, resultados e discussão de artigos científicos. A lista de verificação e os documentos que descrevem a iniciativa STROBE foram elaborados por meio de um processo de colaboração que inclui pesquisadores que trabalham na área de epidemiologia, estatística e metodologia de pesquisa, além de editores de diversas revistas científicas (Malta, Cardoso, Bastos, Magnanini \& Silva, 2010).

Para coleta de dados o instrumento utilizado foi o Índice de Capacidade para o Trabalho (ICT), que é um instrumento que foi traduzido em português e validado no Brasil e sua reprodutibilidade é relevante quanto às propriedades psicométricas. Este instrumento é composto de perguntas abertas e fechadas distribuídas em duas partes: a primeira parte é um questionário de dados gerais dos profissionais de saúde da atenção básica; e a segunda parte é o Índice de Capacidade para o Trabalho propriamente dito, em que o profissional se autoavalia na sua capacidade para o trabalho, no momento atual e em um futuro próximo (Tuomi et al., 1997).

Além de apresentar boa aplicabilidade e demonstrar-se como importante ferramenta ao controle de doenças ocupacionais, o questionário que compõe este instrumento pode sofrer pequenas alterações na linguagem traduzida com a intenção de garantir a intelecção do texto, contudo, deve-se manter o mais próximo possível do original (Silva Junior, Vasconcelos, Griep \& Rotenberg, 2011; Tuomi, Ilmarinen, Jahkola, Katajarinne \& Tulkki, 2010; Tuomi et al., 1997; WHO, 1993).

Para elaboração do instrumento e coleta de dados utilizou-se o aplicativo Magpi, da Internet, que serve para implantar formulários de coleta de dados móveis e transformá-los em banco de dados. Oferece ferramentas para coleta de dados móveis, mensagens e visualizações que permitem reunir informações, gerenciar equipes, obter entendimento, entre outros recursos, que estão disponíveis na Internet. Este instrumento foi aplicado aos 88 profissionais da Estratégia Saúde da Família (ESF), durante a pesquisa realizada no período de julho a novembro de 2018, em nove unidades da Assistência Primária à Saúde (APS), das regiões administrativas: Região Metropolitana II do RJ e Unidades da Região de Saúde: Praias da Baía I e II, que fazem parte da ESF, do município de Niterói, Rio de Janeiro.

A abordagem ocorreu em um encontro apresentando: convite verbal, explicação sobre os propósitos da pesquisa e sobre o questionário. A coleta de dados foi realizada em salas disponibilizadas pelas equipes das Unidades de Saúde participantes. O tempo médio para os participantes responderem todas as perguntas do instrumento foi de vinte (20) minutos e o tempo médio da permanência do pesquisador em cada unidade de saúde foi de quatro (04) horas. Em algumas unidades foi necessária mais de uma visita devido à intensa atividade laboral, no momento da coleta de dados e a falta de disponibilidade da equipe para dispor do tempo mínimo em responder o instrumento de pesquisa. Todas as visitas foram previamente agendadas com o coordenador responsável pelas unidades participantes.

Ressalta-se que as regiões administrativas Baía I e II eram compostas por 11 unidades, com $n=118$ profissionais. Foram excluídos $\mathrm{n}=30$ profissionais, pois 17 não se encontravam nas unidades de ESF durante a pesquisa, por motivos de ausências por férias e/ou licenças médicas. Também foram excluídos 13 profissionais, que eram de duas unidades administrativas excluídas devido à violência urbana e dificuldade de acessibilidade local. Assim foi incluído na pesquisa o total de 88 profissionais.

Para a análise dos resultados e estatística, os dados coletados a partir dos instrumentos de pesquisa foram dispostos em uma planilha eletrônica do programa Microsoft Excel 2013, construindo assim o banco de dados da pesquisa. O programa Microsoft Excel também foi utilizado para construção de alguns gráficos descritivos. Todas as demais análises estatísticas dos 
dados foram feitas através do programa IBM SPSS (Statistical Package for the Social Sciences), versão 22.0 (Pagano \& Gauvreau, 2004).

A análise descritiva dos dados foi realizada descrevendo as variáveis do ICT em relação aos profissionais da ESF, e foi realizada baseada na construção de gráficos, distribuições de frequências e cálculo de estatísticas descritivas (proporções de interesse para todas as variáveis e cálculo de mínimo, máximo, média, mediana, desvio padrão, coeficiente de variação - CV para as variáveis quantitativas). A variabilidade da distribuição de uma variável quantitativa foi considerada baixa se $C V<0,20$, moderada se $0,20 \leq C V<0,40$, e alta se $C V \geq 0,40$. (Pagano \& Gauvreau, 2004).

Foram respeitados os aspectos éticos, em conformidade com a Resolução no 466/2012, sobre Pesquisas Envolvendo Seres Humanos. A pesquisa foi aprovada pelo Comitê de Ética da Faculdade de Medicina/ Hospital Universitário Antônio Pedro - CEP CMM/ HUAP no 143/2010, como parte de uma pesquisa maior intitulada: "Educar para melhor cuidar - uma cartografia das necessidades de saúde de usuários da atenção básica". Caracteriza-se como observacional, do tipo transversal, descritivo norteado pela iniciativa Strengthening the Reporting of Observational Studies in Epidemiology (STROBE, 2007). Foram incluídos na pesquisa 88 profissionais após assinaturas dos Termos de Consentimento Livre e Esclarecido (TCLE).

\section{Resultados e Discussão}

Os resultados foram compilados do instrumento ICT, que gerou dados estatísticos comprobatórios e robustos. Sendo descritos, categorizados e organizados de forma clara, atendendo aos objetivos gerais e específicos da pesquisa epidemiológica. Participaram deste estudo 88 profissionais lotados nas equipes multiprofissionais da Estratégia de Saúde da Família (ESF), da Atenção Primária à Saúde (APS), e sendo 80,7\% dos participantes do gênero feminino. A feminilização das ocupações e profissões de saúde já é uma tendência tanto nacional quanto internacional, como demonstra estudo de Matos, Toassi \& Oliveira (2013), que expuseram a grande participação da mão de obra feminina na área da saúde e também em todas as outras profissões. No entanto, o censo brasileiro demonstrou a desigualdade existente entre os gêneros, pois, apesar de a quantidade de mulheres com ensino superior completo ser consideravelmente maior que a de homens $(24,3 \%$ das mulheres e $14,6 \%$ dos homens), estas recebem $24,4 \%$ a menos que os homens no mercado de trabalho (IBGE, 2019; Saraiva, Bello, \& Renaux, 2018).

Conforme demonstra a Tabela 1, os diversos profissionais que compõem as equipes de saúde desta pesquisa foram avaliados com a utilização do instrumento de avaliação ICT. A área de Baía I concentrou 11,4\% dos profissionais participantes da pesquisa e a de Baía II, 88,6\%.

Tabela 1 - Valor percentual e absoluto dos participantes do estudo segundo categoria profissional. Rio de Janeiro, Brasil, 2018.

\begin{tabular}{ccc}
\hline \hline & & $\begin{array}{c}\text { Global } \\
(\mathbf{n = 8 8})\end{array}$ \\
\cline { 3 - 3 } Tipo de profissional & & $\boldsymbol{\%}$ \\
\hline Recepcionista/Telefonista & 2 & 2,2 \\
Agente Comunitário de Saúde & 29 & 33,0 \\
Cirurgião Dentista & 3 & 3,4 \\
Enfermeiro & 17 & 19,3 \\
Fonoaudiólogo & 1 & 1,1 \\
Médico & 17 & 19,3 \\
Técnico de Enfermagem & 19 & 21,6 \\
\hline
\end{tabular}

Legenda: frequência: n; porcentagem: \%. Fonte: Pesquisa (2018). 
Os profissionais que se destacaram com maior quantitativo foram os agentes comunitários de saúde (ACS) com 29 (33,0\%) participantes, técnicos de enfermagem com 19 (21,6\%), e enfermeiros e médicos, ambos com 17 (19,3\%). Assim como afirma a Política Nacional de Atenção Básica (Brasil, 2017a), a equipe mínima das UBS é composta por: médico generalista, ou especialista em Saúde da Família, ou médico de família e comunidade; enfermeiro generalista ou especialista em Saúde da Família; auxiliar ou técnico de enfermagem; e agentes comunitários de saúde. Podem ser acrescentados a essa composição os profissionais de Saúde Bucal: cirurgião-dentista generalista ou especialista em Saúde da Família; auxiliar e/ou técnico em Saúde Bucal.

Os profissionais da ESF atuam no campo da política pública de saúde, no âmbito do SUS; política essa que se contrapõe à força dos interesses privados, que lutam pelo controle da gestão privada dos serviços públicos. Interesses esses manifestos pela via da terceirização, e/ou mesmo manifesto na trama e na ofensiva de desqualificação de que tudo que é público é, e deve ser ruim, precário, e tudo que é privado é ótimo, competente, enfim, de excelência (Oliveira, Pitta, \& Amarante, 2017).

A ideia pejorativa deve ser combatida, pois o SUS e os serviços públicos têm uma atuação fundamental na vida de todos os brasileiros, e dando acesso inclusive às classes sociais menos favorecidas, que necessitam deste serviço para o cuidado de sua saúde e de seus familiares. É uma política pública recente, ou seja, a saúde é um direito e não uma mercadoria, por isso o SUS deve ser defendido devido a sua ampla atuação, sendo uma questão cultural e de desconstrução do imaginário social de que o serviço público é inferior ao privado. O SUS fundamenta-se em três princípios: "universalidade, igualdade e equidade" (Brasil, 2020). O que necessita é que o orçamento público seja maior para suprir as demandas institucionais, estruturais, de mão de obra, de materiais, de pesquisa, e tudo que possa aprimorar cada vez mais estes serviços.

Outra questão relevante é que os profissionais declararam que se sentiam ameaçados no local de trabalho, devido à violência urbana $(68,2 \%)$ dos profissionais desta pesquisa. Assim é necessário um olhar mais atento das autoridades governamentais e gestores de saúde referente à segurança dos trabalhadores, que exercem suas atividades laborais em áreas de risco devido à violência urbana bem como os usuários das UBS (Silveira, Christovam, Ferreira, Sá \& Pitta, 2020).

A análise dos coeficientes de variação em relação à idade e ao trabalho dos participantes é demonstrada na Tabela 2.

Tabela 2 - Principais estatísticas das distribuições de idade, idade que começou a trabalhar, tempo que trabalha na instituição e carga horária semanal total dos profissionais de saúde que atuavam nas ESF de Niterói, Rio de Janeiro, Brasil, 2018.

\begin{tabular}{c|cccccc}
\hline \hline Variável & Mínimo & Máximo & Média & Mediana & $\begin{array}{c}\text { Desvio } \\
\text { Padrão }\end{array}$ & C.V \\
\hline Idade (anos) & 23 & 66 & 40,6 & 39 & 10,4 & 0,26 \\
Idade que começou a trabalhar (anos) & 11 & 35 & 18,6 & 18 & 4,2 & 0,23 \\
Tempo de trabalho (anos) & 0 & 52 & 22,0 & 20,5 & 10,9 & 0,50 \\
$\begin{array}{c}\text { Tempo de trabalho na instituição } \\
\text { (anos) }\end{array}$ & 0,17 & 25 & 6,7 & 5,5 & 6,7 & 1,00 \\
Carga horaria semanal total (h) & 40 & 74 & 42,4 & 40 & 7,0 & 0,17 \\
\hline
\end{tabular}

Legenda: Coeficiente de Variação - C. V. Fonte: Pesquisa (2018).

Os dados apontam que o grupo de profissionais é homogêneo relacionado à carga horária total semanal $(\mathrm{CV}=0,17<0,20)$, apresenta variabilidade moderada na idade que começou a trabalhar ( $\mathrm{CV}=0,26$ e 0,23 , respectivamente), e é muito heterogêneo no que diz respeito ao tempo de trabalho $(\mathrm{CV}=0,50)$ e tempo que trabalha na instituição $(\mathrm{CV}=1,0)$.

A menor idade foi 23 e a maior foi 66 anos, com média de (40,6\%). A maioria dos participantes apresentou idade de 23 a 49 anos (76,2\%), começou a trabalhar na faixa etária de 15 a 19 anos (42,0\%), e eram casados (37,5\%). Quanto ao nível 
de escolaridade, 39,8\% possuía ensino médio completo, superior ou pós-graduação completa (29,5\%), Quanto às questões de trabalho, os profissionais têm salário típico de 1 a 2 salários mínimos $(51,1 \%)$, não possuem outras atividades de trabalho (85,2\%), trabalham 40 horas por semana no total (87,5\%), têm tempo de experiência de trabalho de 9 a 27 anos $(62,5 \%)$ e trabalham na unidade de saúde há menos de 1 ano $(30,7 \%)$ e de 5 a 9 anos $(25,0 \%)$.

A idade média simples dos participantes da pesquisa foi de 43 anos. Evidenciou-se que a idade em que os profissionais iniciaram suas atividades laborais foi relativamente baixa. Corroborando este resultado demonstrando que quanto menor a renda da família, menor é a idade que os membros iniciam o trabalho remunerado. E o que piora ainda mais a situação, são os dados que mostram que quanto mais cedo se começa a trabalhar, menor será a remuneração desta pessoa no futuro (TRT-4, 2016). A partir de dados da Pesquisa Nacional por amostra de domicílios do Instituto Brasileiro de Geografia e Estatística - IBGE (relativos aos anos de 2001 a 2009 e de 2011), estes estudos comparam rendas de trabalhadores adultos que possuem o mesmo nível de escolaridade, mas ingressaram no mercado de trabalho em idades diferentes.

Todos os profissionais trabalhavam 40 horas por semana na instituição, não recebiam gratificação por insalubridade, não eram concursados, não tinham carteira assinada ou qualquer vínculo empregatício e somente uma médica era terceirizada. Este adicional ainda é regido pela nossa Carta Magna em seu artigo $7^{\circ}$, que define a insalubridade como um direito do trabalhador, assim como a Consolidação das Leis Trabalhistas, CLT, em seus artigos 189 a 192, a qual aduz sobre a responsabilidade do Ministério do Trabalho regulamentar quais atividades serão consideradas insalubres, tais como a caracterização da insalubridade, os limites de tolerância, os meios de proteção e o tempo máximo de exposição (Brasil, 2017b).

$\mathrm{O}$ adicional de insalubridade é um direito concedido aos servidores que trabalham diretamente expostos a agentes nocivos à saúde, como agentes químicos e biológicos, radiações e outros, podendo ser em grau mínimo (10\%) sob o valor do salário, médio (20\%) sob o valor do salário e máximo (40\%) sob o valor do salário (Brasil, 2017b).

A Tabela 3 mostra a distribuição de frequências das principais comorbidades que acometem pelo menos 5\% dos profissionais de saúde.

Tabela 3 - Distribuição de frequências das principais comorbidades que acometem pelo menos 5\% dos profissionais de saúde nas ESF de Niterói, Rio de Janeiro, Brasil, 2018.

\begin{tabular}{|c|c|c|c|c|c|c|}
\hline \multirow[t]{2}{*}{ Comorbidade } & \multicolumn{2}{|c|}{$\begin{array}{l}\text { Diagnosticada } \\
\text { por profissional }\end{array}$} & \multicolumn{2}{|c|}{$\begin{array}{l}\text { Autodiagnosticad } \\
\quad \text { a }\end{array}$} & \multicolumn{2}{|c|}{$\begin{array}{c}(\mathrm{n}=88) \\
\text { Total }\end{array}$} \\
\hline & & $\%$ & $\mathbf{n}$ & $\%$ & & \\
\hline HAS & 23 & 26,1 & - & - & 23 & 26,1 \\
\hline Lesão nas costas & 17 & 19,3 & 6 & 6,8 & 23 & 26,1 \\
\hline Lesão nas Pernas & 17 & 19,3 & 2 & 2,3 & 19 & 21,6 \\
\hline Distúrbio Emocional - Depressão Leve & 13 & 14,8 & 5 & 5,7 & 18 & 20,5 \\
\hline Obesidade & 18 & 20,5 & - & - & 18 & 20,5 \\
\hline Lesão nos Braços/ Mãos & 11 & 12,5 & 3 & 3,4 & 14 & 15,9 \\
\hline Alergia- Eczema & 11 & 12,5 & 3 & 3,4 & 14 & 15,9 \\
\hline Sinusite Crônica & 13 & 14,8 & - & - & 13 & 14,8 \\
\hline Lesão em outras partes do Corpo & 7 & 8,0 & 4 & 4,5 & 11 & 12,5 \\
\hline Diabetes Mellitus & 11 & 12,5 & - & - & 11 & 12,5 \\
\hline Doença na parte superior das costas & 6 & 6,8 & 4 & 4,5 & 10 & 11,4 \\
\hline Asma & 7 & 8,0 & - & - & 7 & 8,0 \\
\hline Dor nas costas que irradia & 2 & 2,3 & - & - & 7 & 8,0 \\
\hline ITUR & 7 & 8,0 & 5 & 5,7 & 7 & 8,0 \\
\hline Doença na parte inferior das costas & 6 & 6,8 & - & - & 6 & 6,8 \\
\hline Duodenal & 5 & 5,7 & 1 & 1,1 & 6 & 6,8 \\
\hline Doença Neurológica -AVC & 4 & 4,5 & 1 & 1,1 & 5 & 5,7 \\
\hline
\end{tabular}

Legenda: frequência: n; porcentagem: \%. Fonte: Pesquisa (2018). 
Os profissionais da pesquisa apresentaram duas ou mais comorbidades crônicas. O número de DCNT variou de 2 a 16, ou seja, todos os profissionais apresentaram comorbidades. Os quantitativos de comorbidades por profissional mais frequentes foram de 2 a $5(65,9 \%)$. A mediana foi de 4 comorbidades, e a média foi de 5,1 comorbidades por profissional. O desvio padrão da distribuição do número de comorbidades foi igual a 3, e resultou no coeficiente de variação igual a 0,59 , e mostrou que o número de comorbidade apresentou alta variabilidade entre os profissionais de saúde.

As comorbidades mais predominantes dentre os profissionais de saúde foram Hipertensão Arterial Sistêmica (HAS) e as lesões nas costas, ambas com $(26,1 \%)$ dos participantes. Também destacam-se as lesões nas pernas com $(21,6 \%)$; a depressão leve e a obesidade com (20,5\%) cada; lesão nos braços/mãos e alergia-eczema com (20,5\%) cada. Confirmando estes resultados, a HAS é identificada como uma doença crônica não transmissível (DCNT), multifatorial relacionada às alterações funcionais, estruturais e metabólicas, e é considerada como um importante problema de saúde pública devido à sua alta prevalência e baixa taxa de controle. Estudos atuais mostraram que a HAS é uma realidade preocupante e que reafirma o papel central da gestão das políticas de saúde estaduais e municipais, que precisam trabalhar na perspectiva intersetorial, no sentido de aprimorar a integralidade da atenção, por meio das estratégias já delineadas nos Planos Nacionais de Controle das Doenças Crônicas, como a Hipertensão arterial, de forma que as ações desenvolvidas possam convergir para as metas estabelecidas (Martins, Abreu, Silva \& Lima, 2020).

Neste estudo as doenças crônicas que se destacaram foram a hipertensão, lesão nas costas, lesão nas pernas, depressão leve e obesidade. Ao passo que nos estudos de Martins et al., (2020), as DCNT mais frequentes foram as doenças cardiovasculares, o diabetes, as neoplasias e as doenças respiratórias crônicas. Portanto, as DCNT necessitam de acompanhamentos contínuos, pois apresentam estados permanentes ou duradouros, e representam um problema de saúde pública relevante, com mais de $70 \%$ das causas de mortes no Brasil, e abrangem principalmente às pessoas com maior idade, sendo o maior motivo de internações hospitalares e de óbitos em idosos (Martins, Abreu, Silva \& Lima, 2020).

As estimativas da Organização Mundial de Saúde (OMS) indicam que as DCNT são responsáveis por $(58,5 \%)$ de todas as mortes ocorridas no mundo e por $(45,9 \%)$ da carga global de doença. Os principais fatores de risco para a HAS são a idade, raça, sexo, sobrepeso ou obesidade e hábitos de vida pouco saudáveis, como sedentarismo, consumo abusivo de bebidas alcoólicas, tabagismo e consumo excessivo de sal. Outros fatores de risco estão associados com a pressão arterial elevada, assim como a predisposição genética e o estresse (Brasil, 2011).

Corroborando esta pesquisa, em relação aos diagnósticos apresentados pelos profissionais, estudos mostraram que a realização do diagnóstico da situação de saúde de determinada população é um dos mais importantes da epidemiologia, e é comumente baseado em dados estatísticos sobre a saúde, ou a sua falta, do conjunto ou de algum seguimento da população. A utilidade da epidemiologia, neste caso, é fornecer orientações e fundamentação necessária para o aprofundamento da análise (Gil, 2002; Pagano \& Gauvreau, 2004).

Referente às exigências do trabalho, dos 88 profissionais que participaram do estudo, 95,5\% declararam que as exigências do trabalho eram físicas e mentais, e somente 4,5\% deles declararam que o trabalho demandava apenas esforço mental, $31,8 \%$ não se sentiam ameaçados no local de trabalho mas a maioria dos profissionais $(68,2 \%)$ declarou que se sentia ameaçado no local de trabalho devido à violência urbana (Silveira, Christovam, Ferreira, Sá \& Pitta, 2020).

$\mathrm{O}$ mundo do trabalho coloca novos desafios à sociedade. Tais desafios comportam reflexões e produção de conhecimento no campo das relações de trabalho que impactam sobre o sofrimento psíquico de trabalhadores e trabalhadoras, particularmente, quando são violados direitos fundamentais e humanos e seus desdobramentos trabalhistas, sanitários, previdenciários entre outros, que garantem a vida sadia e digna (Portero \& Vaquero, 2015).

A exigência física e principalmente a mental, por vezes, podem provocar danos irreparáveis à saúde do trabalhador. Para embasar e fomentar esse tema, conta-se com pesquisadores e especialistas, que incansavelmente buscam uma Política 
Pública de Saúde (PPS) eficiente e real para todos. Concernente ao desgaste emocional oriundo do estresse, pesquisas internacionais, como a realizada em Córdoba, Espanha, apresentaram resultados semelhantes ao deste estudo, em que o desgaste mental pode levar à exaustão emocional (Portero \& Vaquero, 2015).

Nota-se que existem Políticas Públicas voltadas à saúde do trabalhador, porém sua aplicabilidade está muito aquém do que deveria ser. $\mathrm{O}$ fato dos profissionais atuantes na ESP não possuírem vínculos empregatícios, agrava mais esta questão, por não possuírem respaldo legal de afastamento do trabalho por motivo de doença ou para cuidar de um parente próximo, podendo ter o adoecimento físico e mental como consequência.

Quanto às comorbidades foram classificadas em 13 tipos distintos, e foram discriminadas e exibidas na Tabela 4.

Tabela 4 - Distribuição de frequências de comorbidades e por tipo de comorbidade dos profissionais de saúde que atuavam nas UFS de Niterói, Rio de Janeiro, Brasil, 2018.

\begin{tabular}{cccccccc}
\hline \hline Tipo de Comorbidade & \multicolumn{3}{c}{$\begin{array}{c}\text { Diagnosticada } \\
\text { por profissional }\end{array}$} & \multicolumn{2}{c}{$\begin{array}{c}\text { Autodiagnostic } \\
\text { ada }\end{array}$} & \multicolumn{3}{c}{$\begin{array}{c}\text { (n=88) } \\
\text { Total }\end{array}$} \\
& & $\mathbf{1}$ & \% & n & \% & n \\
\hline Problemas musculoesqueléticos & 50 & 56,8 & 9 & 10,2 & 59 & 67,0 \\
(exceto problemas nas costas/colunas) & & & & & & & \\
Problemas nas costas/coluna & 45 & 51,1 & & 10 & 11,4 & 55 & 62,5 \\
Problemas respiratórios & 39 & 44,3 & 3 & 3,4 & 42 & 47,7 \\
Problemas cardiovasculares & 36 & 40,9 & 0 & 0,0 & 36 & 40,9 \\
Doença endócrino ou metabólica & 31 & 35,2 & 0 & 0,0 & 31 & 35,2 \\
Problemas neurológicos & 22 & 25,0 & 6 & 6,8 & 28 & 31,8 \\
Doença de pele & 13 & 14,8 & 10 & 11,4 & 23 & 26,1 \\
Problemas geniturinários & 20 & 22,7 & 0 & 0,0 & 20 & 22,7 \\
Problemas no aparelho digestivo & 17 & 19,3 & 1 & 1,1 & 18 & 20,5 \\
Problemas de audição & 2 & 2,3 & 2 & 2,3 & 4 & 4,5 \\
Problemas de visão & 2 & 2,3 & 0 & 0,0 & 2 & 2,3 \\
Doença de sangue & 2 & 2,3 & 0 & 0,0 & 2 & 2,3 \\
Defeito de nascimento & 1 & 1,1 & 0 & 0,0 & 1 & 1,1 \\
\hline
\end{tabular}

Legenda: frequência: n; porcentagem: \%. Fonte: Pesquisa (2018).

As comorbidades que acometeram mais de $60 \%$ dos profissionais de saúde foram problemas músculoesqueléticos, exceto os problemas nas costas (67\%), e os problemas nas costas/colunas (62,5\%). Observa-se que as DCNT estão consideravelmente associadas à capacidade funcional dos profissionais de saúde da Atenção Primária à Saúde, pois muitos profissionais apresentaram ausência por faltar ao trabalho devido aos problemas de saúde, sendo assim, (30,7\%) tiveram afastamento de até 9 dias do trabalho e, $(9,1 \%)$ foram afastados das atividades laborais de 10 a 24 dias nos últimos 12 meses. No entanto, $(60,2 \%)$ dos profissionais de saúde informaram que não estiveram fora do trabalho nenhum dia por causa de problemas de saúde, consulta médica ou para fazer exame durante os últimos 12 meses. Talvez estes resultados sejam devido as idades dos participantes, pois $(76,2 \%)$ tinham entre 23 a 49 anos e $(23,8 \%)$ tinham entre 49 a 66 anos. Também o fato de não fazerem exames regularmente demonstra descuido com a saúde. Contudo, mesmo com as frequências relativamente altas para algumas comorbidades, nenhum dos profissionais declarou ter lesão/doença como impedimento para realização do trabalho atual.

Assim é relevante que os gestores de saúde apresentem eficácia na aplicabilidade das Políticas Públicas (PP) voltadas para o envelhecimento saudável dos profissionais como um todo, com programas que incentivam à prática de atividades físicas, hábitos saudáveis, como redução do consumo de álcool e tabaco e alimentação saudável, com o propósito de se manter 
a saúde dos indivíduos considerados idosos pela Política Nacional do Idoso. Assim sendo, algumas doenças crônicas poderiam ser evitadas, ou minimizadas, como também controladas em uma unidade de atenção primária à saúde. Conhecer a forma como as pessoas de meia idade percebem a saúde, como vivem o processo de envelhecimento e o que fazem para se manterem saudáveis pode auxiliar no planejamento de políticas públicas que promovam a saúde dos adultos de meia idade e dos idosos (Mari, Alves, Aerts, \& Câmara, 2016).

Os profissionais classificaram sua capacidade atual para o trabalho em relação às exigências físicas e mentais, conforme a distribuição das respostas na Tabela 5 .

Tabela 5 - Classificação dada pelos profissionais de saúde para suas próprias capacidades de trabalho. Niterói, Rio de Janeiro, 2018.

\begin{tabular}{|c|c|c|c|c|}
\hline \multirow{2}{*}{$\begin{array}{c}\text { Classificação da capacidade atual do } \\
\text { trabalho }\end{array}$} & \multicolumn{2}{|c|}{$\begin{array}{l}\text { Exigências físicas do } \\
\text { trabalho }\end{array}$} & \multicolumn{2}{|c|}{$\begin{array}{c}\text { Exigências mentais do } \\
\text { trabalho } \\
(n=88)\end{array}$} \\
\hline & $\mathbf{n}$ & $\%$ & n & $\%$ \\
\hline Baixa & 1 & 1,1 & 0 & 0,0 \\
\hline Moderada & 19 & 21,6 & 11 & 12,5 \\
\hline Boa & 32 & 36,4 & 44 & 50,0 \\
\hline Muito Boa & 36 & 40,9 & 33 & 37,5 \\
\hline
\end{tabular}

Fonte: Pesquisa (2018).

Para ambas as exigências, a mediana das respostas dos profissionais foi de capacidade "moderada" $(21,6 \%)$. A resposta para as exigências físicas foi de "capacidade muito boa" (40,9\%), com considerável frequência para a "capacidade boa" (36,4\%). Para as exigências mentais a classificação típica de destaque foi a "capacidade boa" (36,4\%) para o trabalho. Não há diferença significativa entre as distribuições das classificações para os dois tipos de exigências, p-valor=0,301, do teste qui-quadrado (Pagano \& Gauvreau, 2004).

Ao utilizar o Instrumento ICT foi realizada a pergunta: Considerando sua saúde hoje, você acha que será capaz de, daqui a 2 anos, fazer seu trabalho atual? O resultado obtido foi classificado como Improvável em 2,3\%. Ainda que tenha sido baixa a representatividade, não se considera desprezível por fazer um contraponto com a variável de desfecho deste estudo, que é a diminuição da capacidade para o trabalho, evidenciando a depressão como principal agravante para o adoecimento do profissional de saúde. Logo, percebe-se a falta de acompanhamento e a pouca valorização referente à saúde dos profissionais, pois parece que a prioridade é dada em alcançar metas e na produção. Os desafios nas ações de prevenção e vigilância em saúde do trabalhador têm sido demonstrados ao analisar e estudar os avanços trabalhistas atuais. (APM, 2002; Brasil, 2016; Brasil, 2017).

Na literatura, quanto à avaliação da capacidade para o trabalho, evidenciou-se um conceito, que mesmo sendo da década de 80 , apresenta-se importante na atualidade. Este índice tem sua definição conceitual pautada na perspectiva do trabalhador ao afirmar: "Quão bem está, ou estará, um(a) trabalhador(a) presentemente ou num futuro próximo, e quão capaz ele ou ela podem executar seu trabalho, em função das exigências, de seu estado de saúde e de suas capacidades físicas e mentais" (Tuomi, Ilmarinen, Jahkola, Katajarinne \& Tulkki, 2010).

Quanto aos trabalhadores mais velhos são mais qualificados e produtivos, mas também podem ser os mais vulneráveis devido às doenças crônicas. A tendência futura, devido ao processo de envelhecimento da população em muitos países como o Brasil, deve manter e até mesmo aumentar a participação no trabalho de funcionários mais velhos. Perder a valiosa experiência e conhecimento desses profissionais, que anteriormente eram tidos como indesejáveis para muitas organizações, na atualidade, 
já está sendo repensada. No entanto, falta aplicabilidade de políticas públicas eficazes visando à saúde do trabalhador com o intuito de prevenir doenças ocupacionais evitáveis.

Assim é importante, considerando principalmente as DCNT e a saúde do trabalhador, o investimento das políticas públicas de saúde em direcionar um olhar às prioridades como a promoção do envelhecimento saudável e ativo, além da manutenção da autonomia e da independência das pessoas que envelhecem, incentivando-as em comportamentos saudáveis, e visando utilizar estratégias adequadas para o enfrentamento de DCNT (Martins Et al., 2020). Também é importante o cuidado e controle das doenças ocupacionais dos trabalhadores com mais idade, pois com melhor saúde, a tendência é permanecerem por mais tempo no mercado de trabalho, e postergar a aposentadoria.

Os dados encontrados neste estudo evidenciaram que a avaliação da capacidade para o trabalho possibilita intervir por meio da promoção da saúde, de ações específicas que contribuam para postergar as incapacidades, assim como reabilitar as incapacidades detectadas e melhorar a qualidade de vida dos profissionais. Logo, a temática em questão, apresenta potencial de investigação e possibilidade para estudos futuros.

\section{Considerações Finais}

$\mathrm{O}$ estudo apresentou os trabalhadores da ESF pesquisada, que foram avaliados pelo Índice de Capacidade para o Trabalho (ICT), além de mapear a capacidade para o trabalho desses profissionais de saúde básica, que envolveu os fatores significativamente associados à capacidade funcional dos profissionais de saúde referentes aos problemas crônicos relacionados ao trabalho.

Destacaram-se as doenças relacionadas com problemas musculoesqueléticos, exceto os problemas nas costas (67\%), e os problemas nas costas/colunas (62,5\%), também os distúrbios emocionais/depressão leve, que influenciaram diretamente na capacidade para o trabalho, e repercutiram assim, em escores mais baixos; sendo que o impacto da depressão leve ( $\mathrm{p}$-valor = 0,002 ) foi ainda superior e mais significativo que o impacto das lesões nas costas ( $\mathrm{p}$-valor $=0,013$ ). Assim sendo, as lesões nas costas e a depressão leve emergiram como fatores que influenciaram diretamente a capacidade ao trabalho, ou seja, profissionais que relataram apresentar essas comorbidades tiveram seus ICT diminuídos em relação aos que não manifestaram as mesmas.

A lacuna de conhecimento evidenciada no estudo sugere a importância de novos estudos que busquem corroborar para o entendimento desta problemática relacionada à saúde do trabalhador, a capacidade para o trabalho, o acompanhamento funcional, o processo de envelhecimento, dentre outros temas importantes relacionados a Atenção Básica de Saúde, e em especial a ESF.

É relevante o investimento governamental e empenho de gestores públicos de saúde na ESF, em se tratando da saúde do trabalhador e sua segurança, visando seus direitos trabalhistas, pois a ausência destas necessidades para o trabalhador podem acarretar em adoecimento precoce e consequentemente, a uma diminuição da capacidade para o trabalho, potencializando a depressão.

Um limitador para este estudo foi a dificuldade de acessibilidade aos locais das UBS devido à violência urbana, pois influenciou na redução do quantitativo de participantes da pesquisa por impedir o acesso dos usuários, dos trabalhadores e dos pesquisadores no período da coleta dos dados da pesquisa.

Portanto, busca-se com este estudo contribuir para pesquisas na temática da saúde do trabalhador, no contexto profissional da ESF pela importância do acompanhamento desses trabalhadores, conforme suas comorbidades crônicas e sugere-se aos gestores da saúde a real aplicabilidade do instrumento ICT, que pode ser associado a outros, no intuito de investigar e prevenir doenças ocupacionais, visando promover um envelhecimento funcional saudável, e enfim valorizar os profissionais da ESF e os usuários deste programa, que faz parte do Sistema Único de Saúde. 


\section{Referências}

Brasil. (2006). Ministério da Saúde. Envelhecimento e saúde da pessoa idosa. Ministério da Saúde. http://bvsms.saude.gov.br/bvs/publicacoes /envelhecimento_saude_pessoa_idosa_n19.pdf.

Brasil. (2011). Ministério da Saúde. Hipertensão arterial e diabetes mellitus: morbidade auto referida segundo o VIGTEL, 2009; cadastro de portadores do SIS-HIPERDIA, 2010. Ministério da Saúde. http://arquivos.sbn.org.br/pdf/vigitel.pdf.

Brasil. (2017a). Ministério da Saúde. Portaria nº 2.436, de 21 de setembro de 2017. Aprova a Política Nacional de Atenção Básica, estabelecendo a revisão de diretrizes para a organização da Atenção Básica, no âmbito do Sistema Único de Saúde (SUS). https://bvsms.saude.gov.br/bvs/saudelegis/gm/20 17/prt2436_22_09_2017.html

Brasil. (2017b). Senado Federal. Consolidação das leis do trabalho - CLT e normas correlatas. Senado Federal. http://www2.senado.leg.br/bdsf/bitstream/handle/id/535468/clt_e_normas_correlatas_1ed.pdf.

Brasil. (2018). Lei $n^{o} 13.595$ de 5 de janeiro de 2018. Altera a Lei $n^{\circ} 11.350$, de 5 de outubro de 2006, para dispor sobre a reformulação das atribuições, a jornada e as condições de trabalho, o grau de formação profissional, os cursos de formação técnica e continuada e a indenização de transporte dos profissionais Agentes Comunitários de Saúde e Agentes de Combate às Endemias. https://www2.camara.leg.br/legin/fed/lei/2018/lei-13595-5-janeiro-2018-786068publicacaooriginal-154714-pl.html.

Brasil. (2020, 24 novembro). Ministério da Saúde (MS). Sistema Único de Saúde (SUS): estrutura, princípios e como funciona. https://www.gov.br/saude/ptbr/assuntos/saude-de-a-a-z-1/s/sistema-unico-de-saude-sus-estrutura-principios-e-como-funciona.

Gil, A. C. (2002). Como elaborar projeto de pesquisa (4a ed.). Atlas.

Instituto Nacional do Seguro Social - INSS. (2019). Nova previdência: confira as principais mudanças. https://www.inss.gov.br/nova-previdencia-confira-asprincipais-mudancas/.

Malta, M., Cardoso L. O., Bastos, F. I., Magnanini, M. M. F., \& Silva, C. M. F. P. (2010). Iniciativa STROBE: subsídios para a comunicação de estudos observacionais. Revista de Saúde Pública, 44(3), 559-565. http://dx.doi.org/10.1590/S0034-89102010000300021.

Mari, F. R., Alves, G. G., Aerts, D. R. G. C., \& Câmara, S. (2016). O processo de envelhecimento e a saúde: o que pensam as pessoas de meia-idade sobre o tema. Revista Brasileira de Geriatria e Gerontologia, 19(1), 35-44. https://doi.org/10.1590/1809-9823.2016.14122.

Martins, N. F. F., Abreu, D. P. G., Silva, M. R. S., \& Lima, J. P. (2020). Produção científica da enfermagem acerca das doenças crônicas não-transmissíveis em pessoas idosas: relações com as necessidades de saúde, as prioridades de pesquisa no Brasil e o trabalho da enfermagem. Research, Society and Development, 9(3), e52932336. http://dx.doi.org/10.33448/rsd-v9i3.2336.

Matos, I. B., Toassi, R. F. C., \& Oliveira, M. C. (2013). Profissões e ocupações de saúde e o processo de feminização: tendências e implicações. Athenea Digital, 13(2), 239-244. https://doi.org/10.5565/rev/athenead/v13n2.1119.

Oliveira, W. F., Pitta, A. M. F., \& Amarante, P. (2017). Trabalho, sofrimento psíquico e direitos humanos. In Oliveira, W. F., Pitta, A. M. F., \& Amarante, P. (Orgs.). Direitos humanos e saúde mental (pp. 450-471). Hucitec.

Organização das Nações Unidas - ONU, Santos, A., tradução. (2002, 2003). Plano de ação internacional contra o envelhecimento. Brasília: Secretaria Especial dos Direitos Humanos. http://www.observatorionacionaldoidoso.fiocruz.br/biblioteca/_manual/5.pdf

Pagano, M., \& Gauvreau, K. (2004). Princípios de bioestatística. Pioneira Thomson Learning.

Portero de la Cruz, S., \& Vaquero Abellán, M. (2015). Desgaste profissional, stress e satisfação no trabalho do pessoal de enfermagem em um hospital universitário. Revista Latino-Americana de Enfermagem, 23(3), 543-552. http://dx.doi.org/10.1590/0104-1169.0284.2586.

Saraiva, A., Bello, L., \& Renaux, P. (2018, 10 abril). No dia da mulher, estatísticas sobre trabalho mostram desigualdade. Agência IBGE Notícias. https://agenciadenoticias.ibge.gov.br/agencia-noticias/2012-agencia-de-noticias/noticias/20287-no-dia-da-mulher-estatisticas-sobre-trabalho-mostramdesigualdade.

Silva Junior, S. H. A., Vasconcelos A. G. G., Griep, R. H., \& Rotenberg, L. (2011, junho). Validade e confiabilidade do ICT em trabalhadores de enfermagem. Rio de Janeiro: Cadernos de Saúde Pública, 27(6),1077-1087. https://www.scielo.br/j/csp/a/YGBCJSbGYq8jcCN3bjTh3Bb/?format=pdf\&lang=pt

Silveira, C. G. S., Christovam, B. P., Ferreira, J. B. S., Sá, S. P. C., \& Pitta, A. M. F. (2020). Impacto da violência urbana aos trabalhadores nas Estratégias de Saúde da Família em áreas de risco. Research, Society and Development, 9(9), e792997892. doi: http://dx.doi.org/10.33448/rsd-v9i9.7892.

STROBE Group. (2007). STROBE Statement: strengthening the reporting of observational studies in epidemiology. Strobe checklist: version 4. Bern: University of Bern. https://www.strobe-statement.org/index.php?id=available-checklists.

Tribunal Regional do Trabalho (4. Região) - TRT-4. (2016). Trabalho infantil: pesquisa revela que quanto mais cedo se começa a trabalhar, menor será a remuneração no futuro. Jusbrasil. Disponível em: https://trt-4.jusbrasil.com.br/noticias/348188175/trabalho-infantil-pesquisa-revela-que-quanto-mais-cedo-secomeca-a-trabalhar-menor-sera-a-remuneracao-no-futuro.

Tuomi, K., Ilmarinen, J., Jahkola, A., Katajarinne, L., \& Tulkki, A. (2010). Índice de capacidade para o trabalho. São Carlos: EdUFSCar.

Tuomi, K., Ilmarinen, J., Klockars, M., Nygard, C. H., Seitsamo, J., Huuhtanen, P., Martikainen, R. \& Aalto, L. (1997). Finish research project on aging workers in 1981-1992. Scandinavian Journal of Work, Environment \& Health, 23(Suppl 1), 7-11. http://www.sjweh.fi/show_abstract.php?abstract_id=207.

World Health Organization - WHO. (1993). Aging and working capacity: report of a WHO study group. Geneva: WHO. http://apps.who.int/iris/bitstream/handle/10665/36979/WHO_TRS_835.pdf;jsessionid=EA267C3364C24797907BD27E9C33539F?sequence=1. 